\title{
Radio monitoring with the Ryle Telescope
}

\section{Guy Pooley* Cavendish Laboratory, University of Cambridge, UK}

E-mail: ggp1@cam.ac.uk

For many years the 5-km Telescope, later renamed the Ryle Telescope, has been used in a monitoring campaign for variable radio sources. The 5-km telescope was built as a high-resolution mapping instrument for Galactic and extragalactic sources. In recent years it became the Ryle Telescope, used for Sunyaev-Zel'dovich observations and for other surveys related to CMB observations. The monitoring of variable sources has always been a secondary program. The telescope is a single-frequency system, and for the past 14 years or so it has been used at $15 \mathrm{GHz}$. A substantial upgrade and reconfiguration is now in progress; the telescope now forms part of the AMI array - the Arcminute Microkelvin Imager.

Some of the monitoring observations have been of transient objects such as radio supernovae, GRBs and X-ray binaries like J1118+480 or CI Cam; there are also long sequences of data on a small number of more persistent XRBs, notably Cygnus X-1, Cygnus X-3 and GRS 1915+105. Examples of some of the more unusual events and the long-term sequences are presented.

VI Microquasar Workshop: Microquasars and Beyond September 18-22, 2006

Como, Italy

* Speaker. 


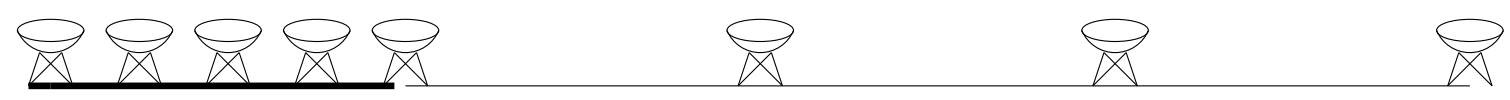

rail track

Figure 1: The layout of the 5-km Telescope. The length of the rail-track and the spacing between the fixed antennas are each $1.2 \mathrm{~km}$.

\section{Introduction}

Radio monitoring of transient and variable sources has been carried out from time to time for many years with what we currently call 'The Ryle Telescope'. I report here on the current state of these observations and try to answer two questions that I have often been asked recently: (a) Why has the regular monitoring stopped? and (b) when will it start again?

\section{History}

To explain the background, we need a short history of the telescope. The 5-km Telescope began operation in 1972, designed and run by a group led by Martin Ryle [1]. It was constructed as an (almost) east-west Earth-rotation synthesis telescope, along the path of a railway which had been closed in 1968 as part of an attempt to stem the financial losses of British Rail. The first purpose-built Earth-rotation radio telescope, the One-Mile Telescope, had been in operation for 10 years on the adjacent site, $8 \mathrm{~km}$ from Cambridge. The telescopes form part of the Mullard Radio Astronomy Observatory, which is operated by the Cavendish Laboratory.

With an Earth-rotation synthesis telescope of this geometry, the majority of mapping observations are $12 \mathrm{~h}$ in duration. Unless you have planned a set of observations with remarkably welldistributed right ascensions, there are inevitably some gaps in the observing schedule which can often be used for monitoring. The great flares of Cygnus X-3 in 1972, which were reported in a whole issue of Nature Physical Science [2], probably alerted us to the possibilities in this area.

The 5-km telescope was designed as a high-resolution mapping instrument. At $5 \mathrm{GHz}$, the initial operating frequency, the resolution was $2 \operatorname{arcsec}$, the best available at that time. It had been designed to achieve an excellent, uniform $u v$ coverage, by using an array with four fixed antennas, $13 \mathrm{~m}$ in diameter, and four (otherwise identical) mobile ones which could be moved along a 1.2-km rail track (not the one abandoned by the railway company!). The geometry is shown in fig 1. It is a single-frequency instrument, and has been operated at 2.7, 5, 15 and (briefly) $31 \mathrm{GHz}$, with resolutions between 3.6 and 0.3 arcseconds.

The distances between the fixed antennas are all $1.2 \mathrm{~km}$, and by using 8 separate configurations of the mobile antennas it was possible to make extraordinarily good images, such as that of Cas A shown in fig 2. (Note that the $u v$ cover is so good that this image does not need cleaning: in AIPS terms it is the 'dirty map'.)

The telescope was used for a long series of mapping observations of extragalactic and galactic sources (e.g. [4]). It gave us the first really clear understanding of the structures of radio sources and led to the development of the beam models of radio galaxies (e.g. [5, 6]).

As a mapping instrument, there were some shortcomings. To change the observing frequency involved changing feed assemblies and took some days. Since the full field of view was only 


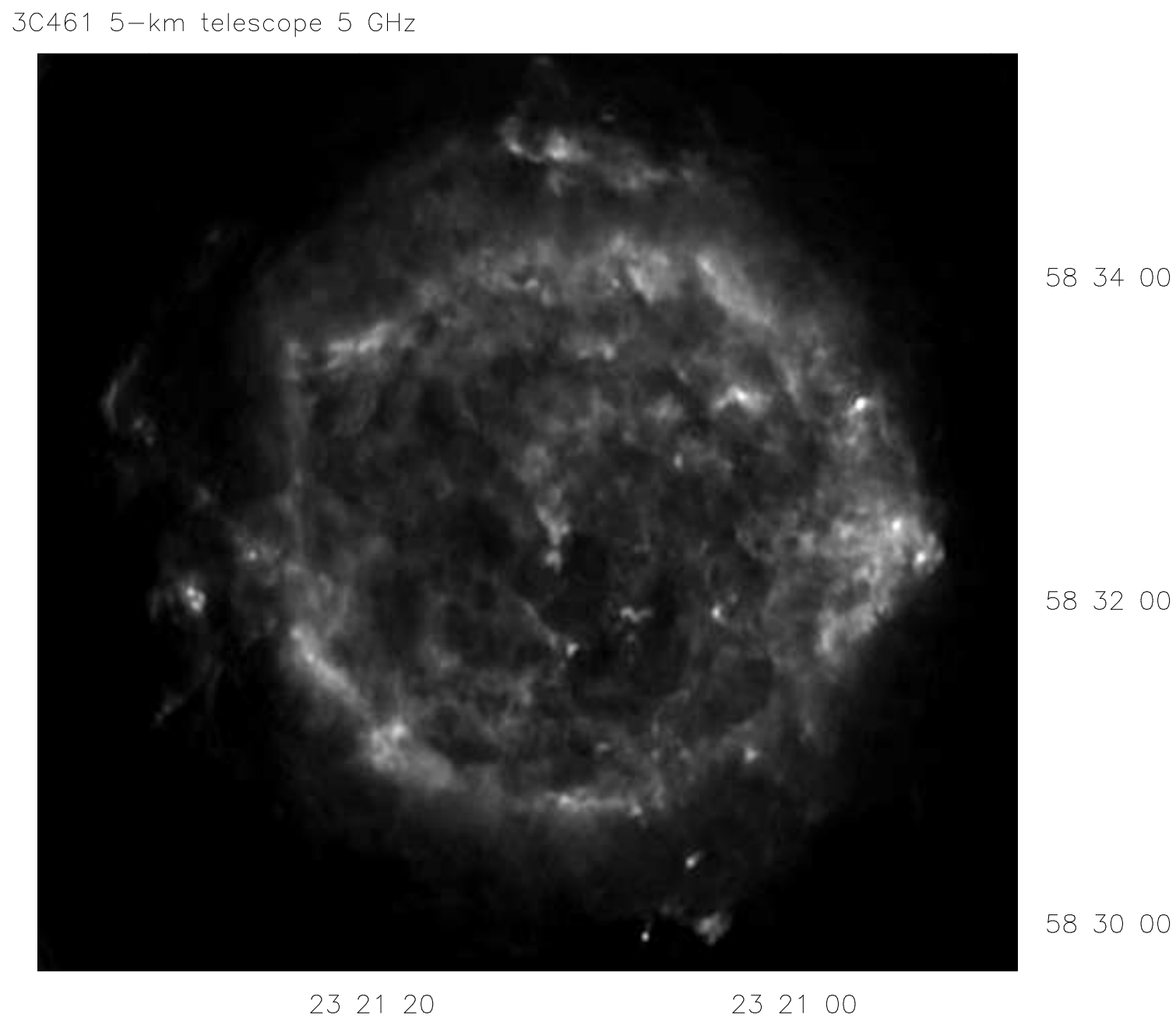

Figure 2: The supernova remnant Cas A, observed with the 5-km telescope at a resolution of 2 arcsec

available if the mobile antennas were used in several configurations, the instrument was not fast in observing (though it was much faster than its predecessor, the One-Mile Telescope). And the excellent aperture coverage was lost when observing near the equator, in common with other eastwest systems (the resolution in declination is proportional to $1 / \sin \delta$ ).

In the early 1980s the VLA came on-line. The improved speed of observation, sensitivity and frequency flexibility offered meant that the $5-\mathrm{km}$ telescope lost its leading position. However, it was realised that it had a valuable role to play in observations of large-scale structures, requiring more modest resolutions around 1 arcmin. From the outset, the rail-track of the telescope had been constructed with some additional parking positions at short baselines. A refurbishment of the system was undertaken [3] involving cryogenic receivers, a (relatively) broad-band ( $350 \mathrm{MHz})$ digital correlator, and the use of an array of baselines up to about $100 \mathrm{~m}$ by parking the mobile antennas close to the first fixed antenna. This is the system that we renamed the Ryle Telescope (fig 3). The 3 antennas at $>1 \mathrm{~km}$ baselines were, after some years, no longer used.

What were the observing programs for the Ryle Telescope? The prime aim was to map the Sunyaev-Zel'dovich (S-Z) effect in clusters of galaxies, which is the result of scattering of the 


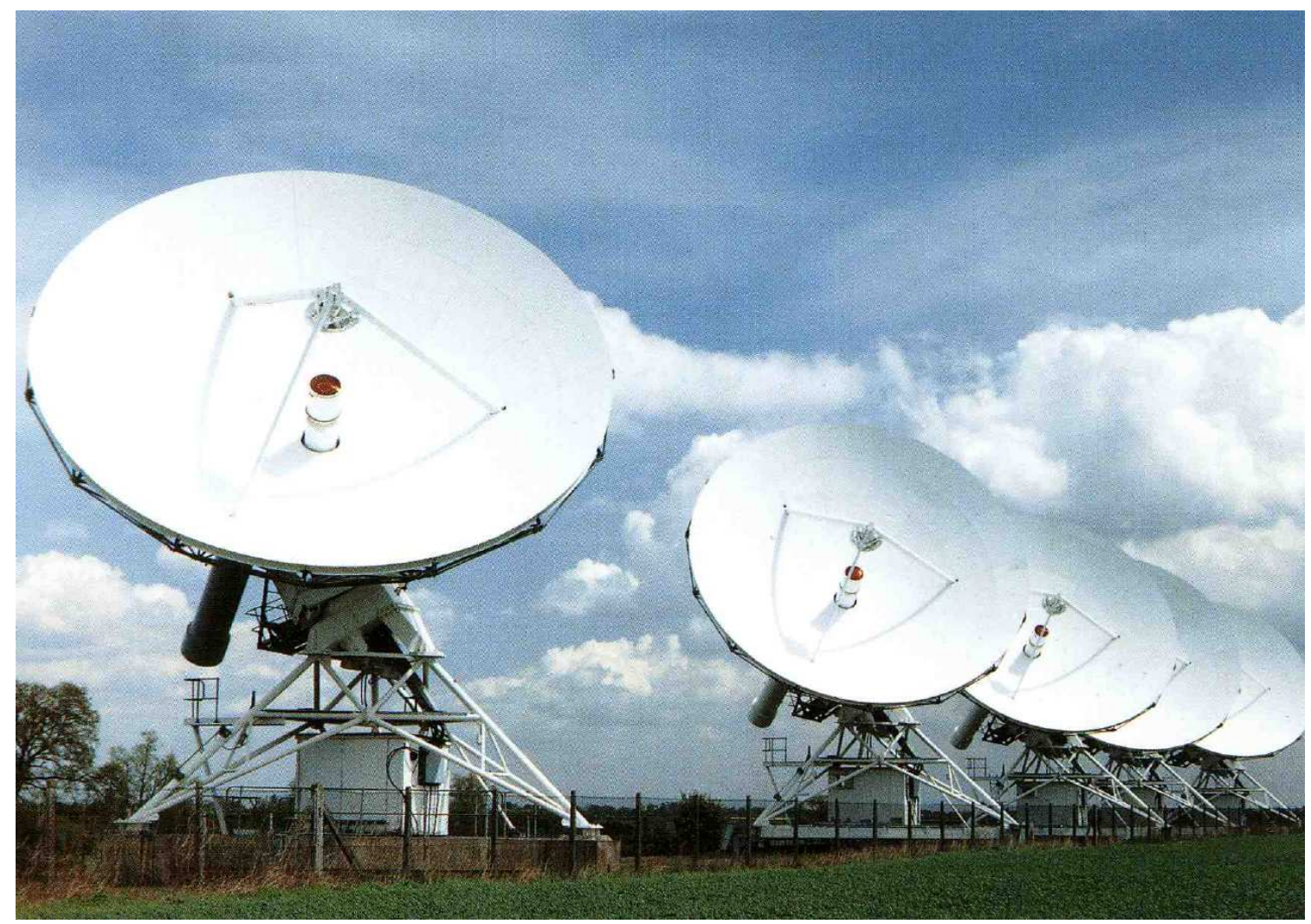

Figure 3: The compact array of the Ryle Telescope

microwave background by the $10^{6} \mathrm{~K}$ gas. In addition, we were operating the pioneering Cosmic Anisotropy Telescope (CAT: [7]) at Cambridge, to map the primordial fluctuations in the background. One of the biggest contaminants for those observations is the presence of radio galaxies and quasars in the foreground. By using a rastering technique the Ryle Telescope was used to map these foreground sources, and we have continued to do the same for the Very Small Array (VSA: [8]). It seemed a waste to merely record these sources and then discard them, so we have (as is traditional in Cambridge radio astronomy) made a catalogue - the 9C catalogue [9] - as a valuable by-product.

\section{The Arcminute Microkelvin Imager (AMI)}

The Ryle Telescope made important Sunyaev-Zeldovitch observations (e.g. [11]), but even in the 'compact array' configuration its resolution is not well matched to the angular size of typical clusters of galaxies (a few arcminutes). To make good S-Z observations, smaller antennas are needed, and we have built an array of these: AMI has ten 4-m antennas, also working at $15 \mathrm{GHz}$. Because of some fortunate astrophysical coincidences the sensitivity of such an instrument is almost independent of redshift, unlike measurements of the X-ray (free-free) emission from the same clusters where the flux falls rapidly with $z$. So it will be possible to make blind surveys for clusters at very high redshifts. As with CAT and the VSA, though, foreground effects are important and we need to survey the same areas, at the same frequency and with the same polarization, to catalogue 
the radio galaxies and quasars. We need only a modest improvement in resolution over the AMI $\mathrm{S}-\mathrm{Z}$ array, but we need to make the observations more-or-less simultaneously (since the foreground sources are often highly variable [10]). We need to make the observations quickly in order to keep up with the small array, so more sensitivity is required.

In order to undertake these observations, we have undertaken a major refit and reconstruction of the Ryle Telescope array. The three distant antennas have been moved to new positions just north of the existing compact array, so we now have some useful resolution at the equator, and can measure signals on more baselines at one time. Front-end amplifiers are being upgraded, the correlator has been replaced with a broad-band system $(6 \mathrm{GHz}$ wide, covering $12-18 \mathrm{GHz})$, with all the consequential changes that are implied. In addition the drive systems and computers are being updated.

This, of course, involved turning off the old system - in late June 2006. We hope to be on the air again in early 2007, although initially with an incomplete system.

We hope to be able to continue the monitoring program.

\section{Highlights of the monitoring program}

What are the monitoring programs that we have been carrying out, and what have we learned from them? For the past 14 years or so we have observed exclusively at $15 \mathrm{GHz}$, and so nearly all the monitoring I describe is at that frequency (there are some earlier data on Cygnus X-3 in particular at 2.7 and $5 \mathrm{GHz}$ ).

Some of the interesting objects are transients of various types (SN1993J, CI Cam, J1118+480, GRB 030329) $[12,13,14,15]$. Observations of these objects are frequently made in collaboration with colleagues around the world: broad-band spectra are often valuable in the interpretation, as is the ability to make prompt observations.

As we have heard in this meeting, the persistent XRB sources are of wide interest. I have concentrated observations on three in particular, Cygnus X-1, Cygnus X-3 and GRS 1915+105. There are also observations of LSI $+61^{\circ} 303$ [16], SS 433 and others, often as a result of specific requests.

\subsection{Cygnus X-3}

Cygnus X-3 has a number of very unusual features. In the radio band it usually has a flux density of around $100 \mathrm{mJy}$. It is subject to enormous outbursts at unpredictable intervals, with the peaks near $15 \mathrm{Jy}$ at $15 \mathrm{GHz}$. There is evidence [17] of a relativistic jet of which the axis is, at least some of the time, directed close to the line of sight. Before the outbursts, there is often a period of 'quenched' emission, when the flux density falls to somewhere near $1 \mathrm{mJy}$. Fig 4 shows the radio flux density for the first 6 months of 2006 (by comparison, 2005 was rather dull).

The periods of quenching can be used to alert observers to a possible outburst. It appears that the longer the period of quenching, the more likely it is that the outburst will be substantial. We do not yet understand enough of the physics of these phenomena. 


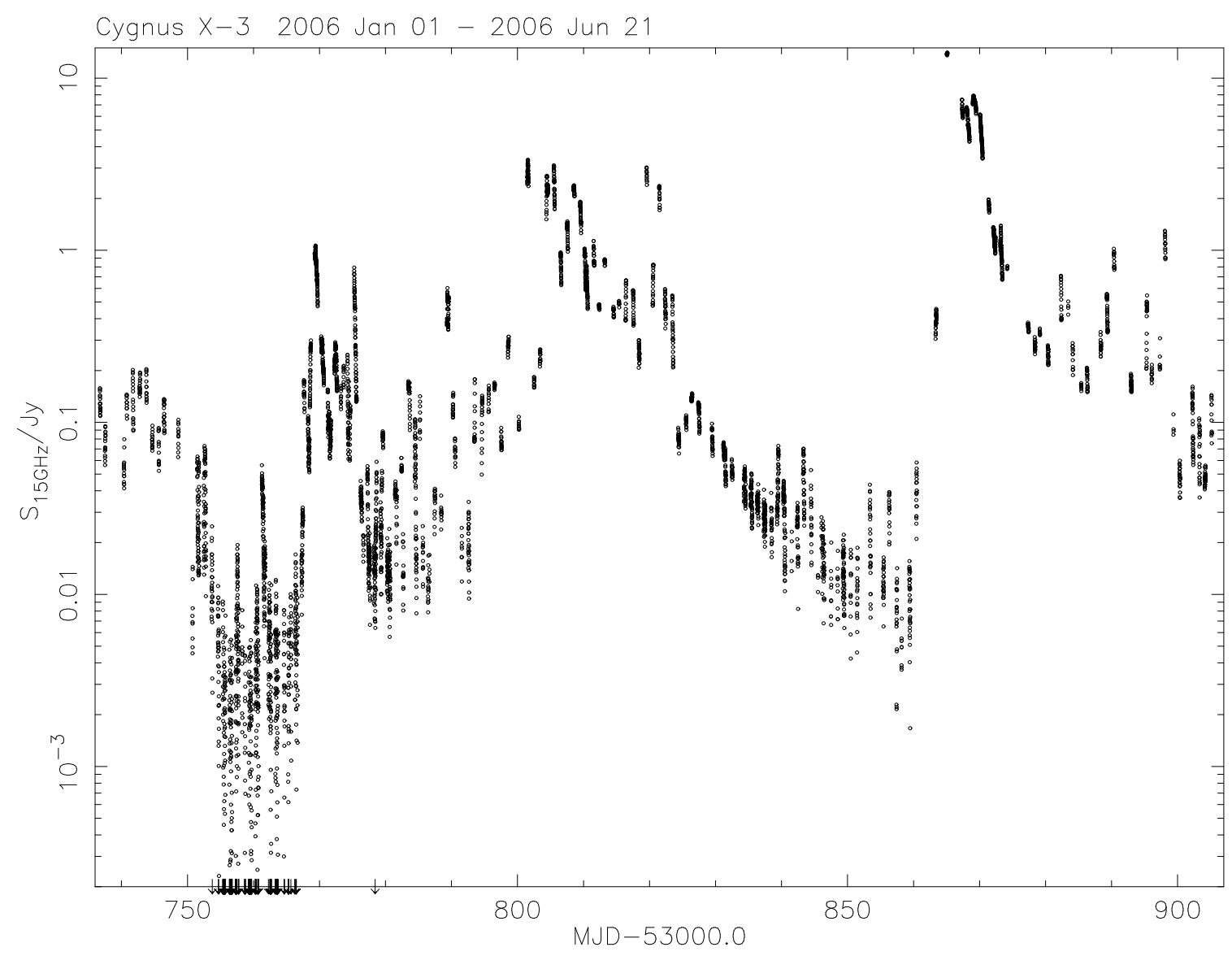

Figure 4: Ryle Telescope observations of Cygnus X-3 during the first half of 2006

\subsection{Cygnus $X-1$}

Cygnus X-1 is perhaps the best-studied of the X-ray binaries, at least in the optical and X-ray bands. Its radio emission is more modest, usually near $15 \mathrm{mJy}$. We found that the radio emission has a component modulated at the binary period $\left(5^{\mathrm{d}} .6\right)$, and there is a suggestion of longer-term variations, around $150^{\mathrm{d}}[18,19]$. When the $\mathrm{X}$-ray state changes to 'high soft', the radio emission falls to a barely-detectable level. We have made a long series of observations coordinated with RXTE (e.g. [20]) as part of a major long-term study of this system.

It is often thought that Cyg X-1 does not show the bright radio flares associated with Cyg X-3, for example. There are flares on Cyg X-1, not as spectacular and very much rarer (I have only seen two or three in 10 years, but they are very short-lived and might well be missed). One specimen is shown in fig 5.

\subsection{GRS 1915+105}

For a transient source, first detected in 1992, GRS 1915+105 is very persistent! Fig 6 illustrates some of the complexity of the radio and X-ray variability, and their relationship. The radio flux density varies on timescales between a few minutes (and probably shorter) and many months. This is a good place to emphasise that the regular monitoring of many sources by the All-Sky Monitor 


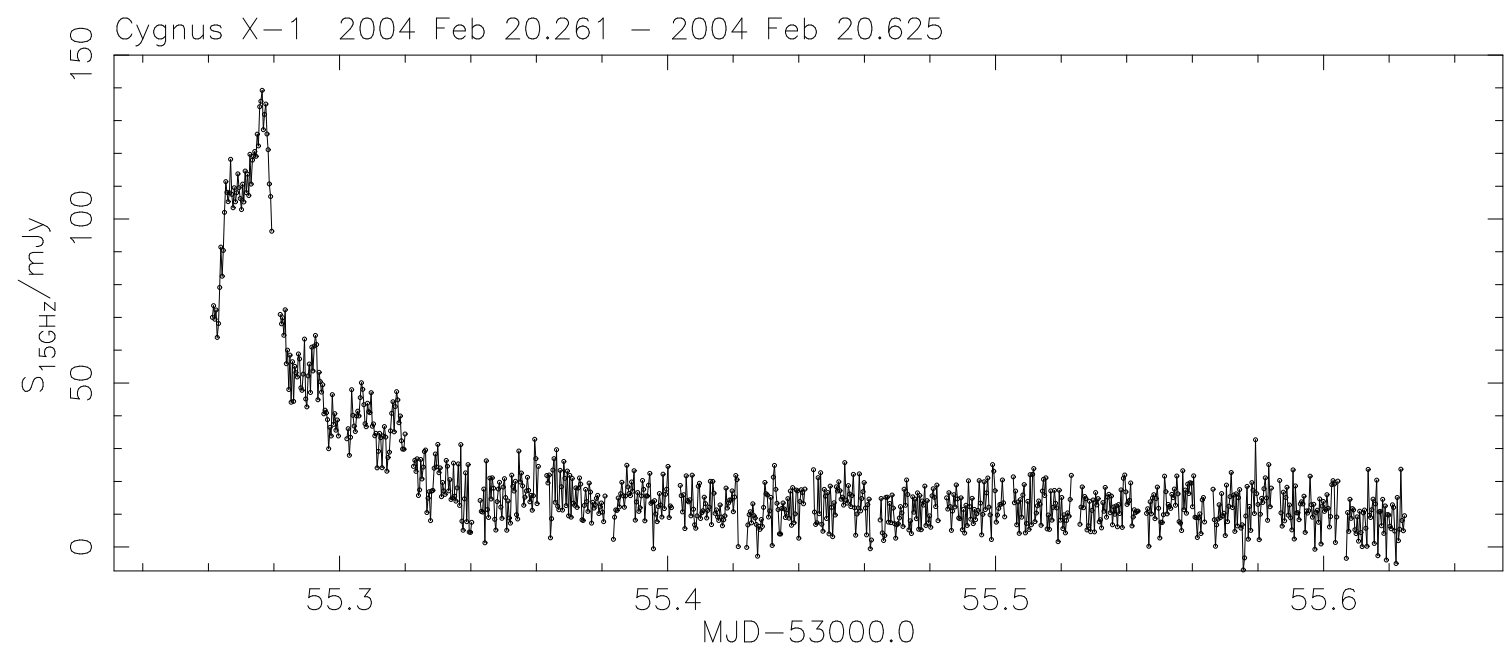

Figure 5: A short-lived 15-GHz radio flare on Cygnus X-1

(ASM) on the RXTE satellite, both in intensity and spectrum (or hardness ratio, in X-ray language) adds greatly to the value of the radio monitoring data.

As in the case of Cygnus X-3, we have used the total-flux monitoring to give alerts of possible relativistic ejections so that MERLIN or VLBI mapping may be triggered (e.g. [21]).

One of the most startling discoveries in the radio monitoring was that of the quasi-periodic oscillations at about $20 \mathrm{~m}$ period [22] (fig 7), which were soon shown to be closely connected to the 'X-ray dips'. We also found similar variations in the infra-red and the mm regimes, e.g. [23], strongly suggesting that the synchrotron emission extends at least into the infra-red band.

\section{Acknowledgments}

The Staff of the Mullard Radio Astronomy Observatory have maintained and upgraded the Ryle Telescope over many years. It has been supported by PPARC and its predecessors. The X-ray data were provided by the ASM/RXTE team at MIT and the Goddard Space Flight Center.

\section{References}

[1] Ryle, M. The 5 km Radio Telescope at Cambridge Nature 239, 435 (1972)

[2] Special issue on Cygnus X-3 Nature Phys Sci 239, No 95, 113-136 (1972)

[3] Jones, M.E. Microwave background interferometry in Cambridge in IAU Colloq. 131 'Interferometry: Theory Techniques \& Applications' ed. T. Cornwell \& R. Perley (ASP conference series No. 19), p395 (1990)

[4] Jenkins, C.J., Pooley, G.G., Riley, J.M. Observations of 104 extragalactic radio sources with the Cambridge 5-km telescope at $5 \mathrm{GHz}$ MemRAS 84, 61 (1977)

[5] Scheuer, P.A.G. Models of extragalactic radio sources with a continuous energy supply from a central object MNRAS 166, 513 (1974)

[6] Blandford, R.D., Rees, M.J. A 'twin-exhaust' model for double radio sources MNRAS 169, 395 (1974) 


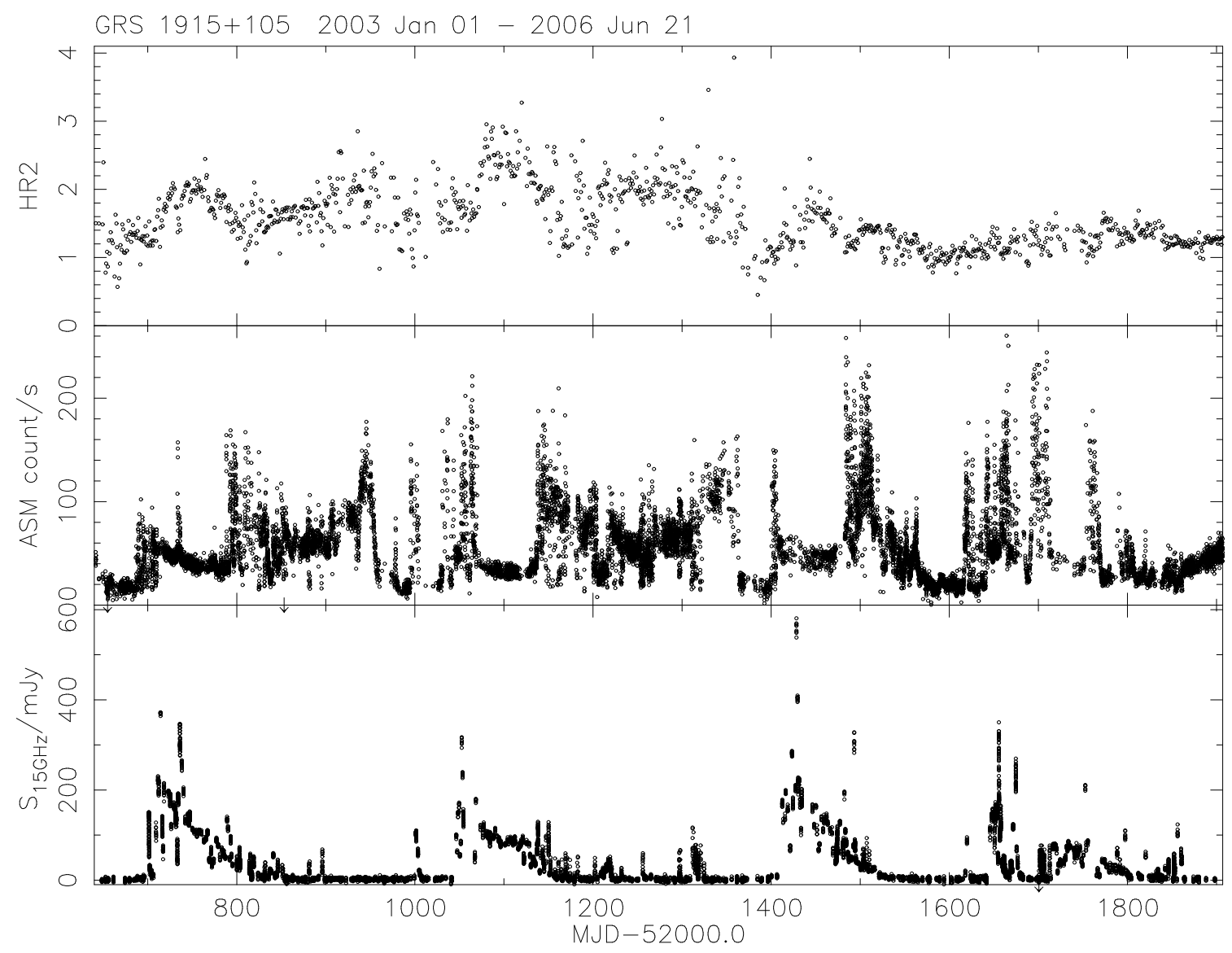

Figure 6: Over 3 years of data from RXTE and the Ryle Telescope for GRS 1915+105

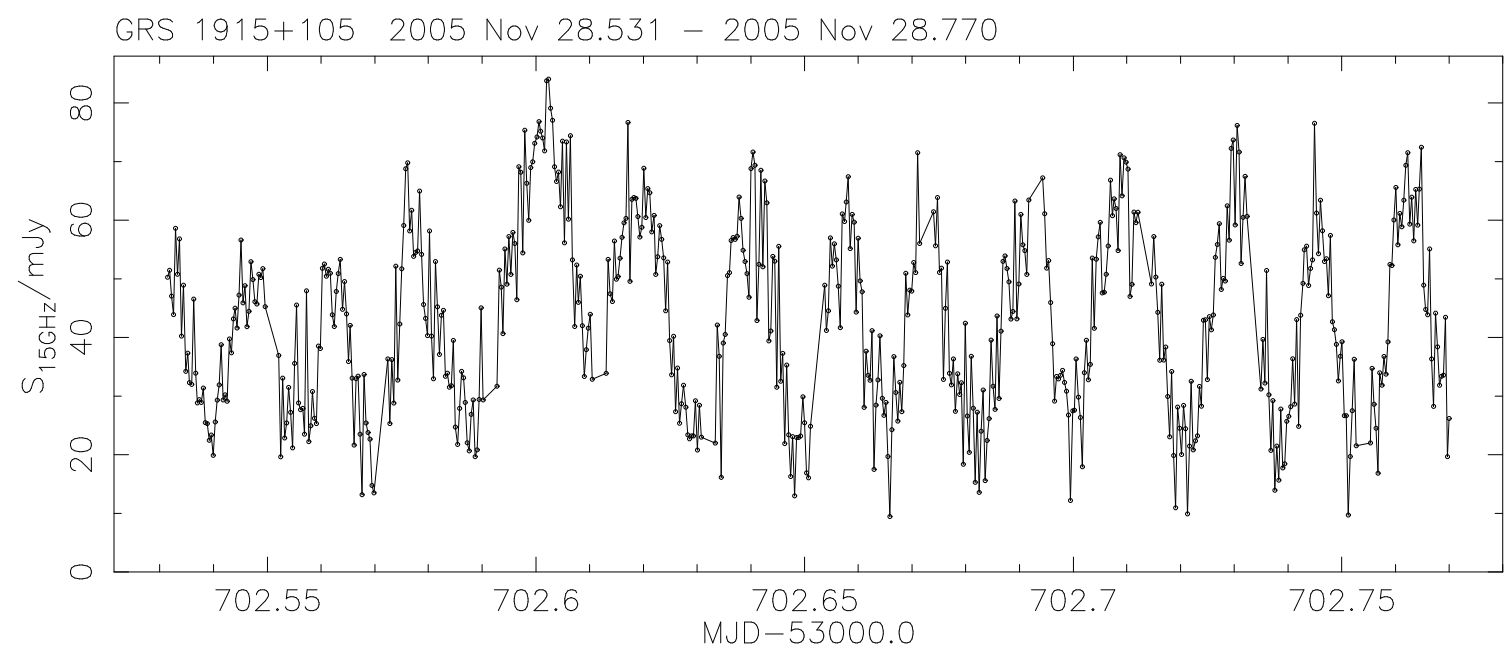

Figure 7: Rapid oscillations of the radio flux density at $15 \mathrm{GHz}$ which are closely coupled with 'X-ray dips' and infrared variations. 
[7] Robson, M. et al. The cosmic anisotropy telescope A\&A 277, 314 (1993)

[8] Watson, R.A. et al. First results from the Very Small Array - I. Observational methods MNRAS 341, 1057 (2003)

[9] Waldram, E.M. et al. 9C: A survey of radio sources at 15GHz with the Ryle Telescope MNRAS 342, 915 (2003)

[10] Bolton, R. C. et al. 15-GHz variability of 9C sources MNRAS 370, 1556 (2006)

[11] Grainge, K. et al. A resolved image of the Sunyaev-Zel'dovich effect in Abell 1413 MNRAS 278, L17 (1996)

[12] Pooley, G.G., Green, D.A. Ryle telescope observations of SN1993J at 15 GHz: The first 115 days MNRAS 264, L17 (1993)

[13] Clark, J.S. et al. Photometric observations of the radio bright B[e]/X-ray binary CI Cam A\&A 356, 50 (2000)

[14] McClintock, J.E. et al. Complete and simultaneous spectral observations of the black-hole candidate XTE J1118+480 ApJ 555, 477 (2001)

[15] Berger, E. et al. A common origin for cosmic explosions inferred from calorimetry of GRB030329 Nature 426, 154 (2003)

[16] Albert, J. et al. Variable very-high-energy gamma-ray emission from the microquasar LS I+61 303 Science 312, 1771 (2006)

[17] Mioduszewski, A.J. et al. A one-sided, highly relativistic jet from Cygnus X-3 ApJ 553, 766 (2001)

[18] Pooley, G.G., Fender, R.P., Brocksopp, C. Orbital modulation and longer-term variability in the radio emission from Cygnus X-1 MNRAS 302 L1 (1999)

[19] Lachowicz, P., Zdziarski, A.A., Schwarzenberg-Czerny, A., Pooley, G.G., Kitamoto, S. Periodic long-term X-ray and radio variability of Cygnus X-1 MNRAS 368, 1025 (2006)

[20] Wilms, J., Nowak, M.A., Pottschmidt, K., Pooley, G.G., Fritz, S. Long-term variability of Cygnus X-1: IV Spectral evolution 1999-2004 A\&A 447, 245 (2006)

[21] Fender, R.P. et al. MERLIN observations of relativistic ejections from GRS 1915+105 MNRAS 304, 865 (1999)

[22] Pooley, G.G., Fender, R.P. The variable radio emission from GRS 1915+105 MNRAS 292, 925 (1997)

[23] Fender, R.P., Pooley, G.G. Giant repeated ejections from GRS 1915+105 MNRAS 318, L1 (2000) 